\title{
Connective tissue growth factor produced by cancer-associated fibroblasts correlates with poor prognosis in epithelioid malignant pleural mesothelioma
}

\author{
YUUKI OHARA $^{1,2}$, ATSUSHI ENOMOTO $^{3}$, YUTA TSUYUKI ${ }^{3}$, KOTARO SATO $^{1}$, TADASHI IIDA $^{3}$, \\ HIROKI KOBAYASHI $^{3}$, YASUYUKI MIZUTANI ${ }^{3}$, YUKI MIYAI ${ }^{3}$, AKITOSHI HARA $^{3}$, SHINJI MII ${ }^{3}$, \\ JUN SUZUKI $^{2}$, KYOKO YAMASHITA ${ }^{1}$, FUMIYA ITO ${ }^{1}$, YASHIRO MOTOOKA ${ }^{1}$, NOBUAKI MISAWA ${ }^{1}$, \\ TAKAYUKI FUKUI ${ }^{4}$, KOJI KAWAGUCHI ${ }^{4}$, KOHEI YOKOI $^{4}$ and SHINYA TOYOKUNI ${ }^{1,5}$ \\ ${ }^{1}$ Department of Pathology and Biological Responses, Nagoya University Graduate School of Medicine, \\ Nagoya 466-8550; ${ }^{2}$ Division of Pathology and Molecular Diagnosis, National Cancer Center Hospital East, \\ Kashiwa 277-8577; Departments of ${ }^{3}$ Pathology and ${ }^{4}$ Thoracic Surgery, Nagoya University Graduate School of Medicine, \\ Nagoya 466-8550, Japan; ${ }^{5}$ Sydney Medical School, The University of Sydney, Sydney, NSW 2006, Australia
}

Received October 24, 2019; Accepted April 29, 2020

DOI: $10.3892 / o r .2020 .7669$

\begin{abstract}
Malignant mesothelioma is an aggressive neoplasm for which effective treatments are lacking. We often encounter mesothelioma cases with a profound desmoplastic reaction, suggesting the involvement of cancer-associated fibroblasts (CAFs) in mesothelioma progression. While the roles of CAFs have been extensively studied in other tumors and have led to the view that the cancer stroma contains heterogeneous populations of CAFs, their roles in mesothelioma remain unknown. We previously showed that connective tissue growth factor (CTGF), a secreted protein, is produced by both mesothelioma cells and fibroblasts and promotes the invasion of mesothelioma cells in vitro. In this study, we examined the clinical relevance of CAFs in mesothelioma. Using surgical specimens of epithelioid malignant pleural mesothelioma, we evaluated the clinicopathological significance of the expression of
\end{abstract}

Correspondence to: Dr Yuuki Ohara or Professor Shinya Toyokuni, Department of Pathology and Biological Responses, Nagoya University Graduate School of Medicine, 65 Tsurumai-cho, Showa-ku, Nagoya 466-8550, Japan

E-mail: yuuki.oohara.1196@gmail.com

E-mail: toyokuni@med.nagoya-u.ac.jp

Abbreviations: $\quad \alpha \mathrm{SMA}, \alpha$-smooth muscle actin; CAFs, cancer-associated fibroblasts; CTGF, connective tissue growth factor; DAB, 3,3'-diaminobenzidine; H\&E, hematoxylin and eosin; IHC, immunohistochemistry; ISH, in situ hybridization; Meflin, mesenchymal stromal cell- and fibroblast-expressing Linx paralogue; ROS, reactive oxygen species

Key words: connective tissue growth factor, CTGF, cancerassociated fibroblasts, tumor microenvironment, malignant mesothelioma, mesenchymal stromal cell- and fibroblast-expressing of a Linx paralogue, Meflin, ISLR, molecular target therapy $\alpha$-smooth muscle actin ( $\alpha \mathrm{SMA})$, the most widely used marker of CAFs, the expression of CTGF, and the extent of fibrosis by immunohistochemistry and Elastica-Masson staining. We also analyzed the expression of mesenchymal stromal cell- and fibroblast-expressing Linx paralogue (Meflin; ISLR), a recently reported CAF marker that labels cancer-restraining CAFs and differ from $\alpha$ SMA-positive CAFs, by in situ hybridization. The extent of fibrosis and CTGF expression in mesothelioma cells did not correlate with patient prognosis. However, the expression of $\alpha \mathrm{SMA}$ and CTGF, but not Meflin, in CAFs correlated with poor prognosis. The data suggest that $\mathrm{CTGF}^{+}$ CAFs are involved in mesothelioma progression and represent a potential molecular target for mesothelioma therapy.

\section{Introduction}

Malignant mesothelioma is a tumor that is primarily caused by exposure to asbestos, including crocidolite, amosite, and chrysotile (1). Mesothelioma is one of the most lethal tumors, with an expected median survival time of 4-18 months for pleural forms (2). There are three main histological types of mesothelioma: Epithelioid, biphasic and sarcomatoid (2). Clinically, patients with the sarcomatoid subtype have the poorest prognosis (3). The molecular mechanisms of asbestos-induced mesothelial carcinogenesis have been recently revealed to include oxidative stress, chronic inflammation, molecular adsorption, and chromosome tangling (4-8). It is necessary to understand the molecular mechanisms that regulate mesothelial carcinogenesis (9-14) and to develop molecular-targeted drugs (15) to improve the prognosis of patients.

Mesothelioma often features a profound desmoplastic reaction since asbestos can develop fibrotic diseases before mesothelial carcinogenesis $(1,2,11)$, suggesting the involvement of cancer-associated fibroblasts (CAFs) in its progression. CAFs occupy the majority of the area in the tumor stroma and produce extracellular matrix (16-20). One of the most well-known CAF markers is $\alpha$-smooth muscle actin ( $\alpha \mathrm{SMA})$. In 
general, CAFs have been shown to exert protumorigenic effects by promoting cancer cell proliferation and invasion. However, recent studies have shown that CAFs are heterogeneous, and the existence of a type of CAF with antitumor functions has been proposed (19-23). Studies of CAF heterogeneity have led to the proposal of multiple CAF markers (16-23). Connective tissue growth factor (CTGF) is known as a protumorigenic CAF marker (18-20). CTGF is a $36-38 \mathrm{kDa}$ multifunctional secretory protein involved in various functions, including cell proliferation, cell invasion and myofibroblast differentiation. We previously demonstrated that CTGF expression is correlated with the malignant behavior of mesothelioma cells (13) and that a CTGF-specific monoclonal antibody (FG-3019, pamrevlumab), which is currently under clinical trials for idiopathic pulmonary fibrosis $(24,25)$ and pancreatic ductal adenocarcinoma (26), was found to inhibit mesothelioma growth (15). Interestingly, we found that CTGF is expressed in both mesothelioma cells and CAFs (15). In contrast, mesenchymal stromal cell- and fibroblast-expressing Linx paralogue (Meflin; ISLR) is a glycosylphosphatidylinositol (GPI)-anchored membrane protein, which has been identified as a marker of mesenchymal stem cells and tissue-resident fibroblasts $(27,28)$. The results of our recent study suggest that Meflin is a potential new marker of antitumorigenic CAFs (29).

Although the functions and heterogeneity of CAFs have been recognized in other tumors, those in the mesothelioma microenvironment have not yet been addressed. We aimed to understand the significance of stromal remodeling during mesothelioma progression. In the present study, we examined the correlations between patient prognosis and the extent of fibrosis, the expression of CAF markers ( $\alpha$ SMA, CTGF and Meflin), and the expression of a cell proliferation marker (Ki-67).

\section{Materials and methods}

Patients. A total of 37 patients underwent surgery for malignant pleural mesothelioma at the Nagoya University Hospital between January 2007 and December 2016. All patients were reviewed for age, sex, histological subtype, pathological invasion (pT), lymph node metastasis $(\mathrm{pN})$, and neoadjuvant therapy. Tumor classification was performed based on the TNM Classification of Malignant Tumors (UICC) 7th edition (30). Patients who had another cancer, who had undergone several surgeries, or who had undergone only cytoreductive surgery were excluded. Based on histological and immunohistochemical analyses, patients with the biphasic or sarcomatoid subtype were also excluded because of the difficulty in distinguishing the mesothelioma cells from CAFs. In total, 22 samples were ultimately analyzed. Human mesothelioma tissues were obtained with informed patient consent at the time of surgery at Nagoya University Hospital (Nagoya, Japan). This study was carried out in accordance with the principles of the Helsinki Declaration for human research and approved by the Ethics Committee of Nagoya University Graduate School of Medicine (protocol no. 2017-0127).

Histologic and immunohistochemical analysis. Four-micronthick serial sections were cut from formalin-fixed and paraffin-embedded tissue and were stained with hematoxylin and eosin (H\&E) or Elastica-Masson or for immunohistochemistry (IHC). The following antibodies were used for
IHC: Anti-CTGF (goat polyclonal, dilution 1:50; Santa Cruz Biotechnology, Inc.; cat. no. 14939), anti-AE1/AE3 (mouse monoclonal, dilution 1:100; Biocare Medical; cat.no.ACR011A, B, C), anti-Ki-67, clone SP6 (rabbit monoclonal, dilution 1:100; Abcam; cat. no. 16667), and anti- $\alpha$ SMA (mouse monoclonal, dilution 1:50; Dako; cat. no. M0851). High-temperature antigen retrieval for CTGF and $\mathrm{Ki}-67$ was performed using Immunosaver (Nisshin EM, Tokyo, Japan) and that for $\alpha \mathrm{SMA}$ and AE1/AE3 was performed using $10 \mathrm{mM}$ Tris (hydroxymethyl) aminomethane/ $1 \mathrm{mM}$ ethylenediaminetetraacetic acid (TE) buffer, $\mathrm{pH}$ 9.0. Following antigen retrieval, the sections were dipped for $30 \mathrm{~min}$ in methanol containing $\mathrm{H}_{2} \mathrm{O}_{2}(0.3 \%$ $\mathrm{vol} / \mathrm{vol}$ ) to quench endogenous peroxidase activity and subsequently blocked with Protein Block Serum-Free Ready-to-use (Dako). For CTGF staining, the avidin-biotin complex method using peroxidase was employed, as previously described (31). For AE1/AE3, Ki-67, and $\alpha$ SMA staining, Histofine Simple Stain MAX-PO (Multi; Nichirei, Tokyo, Japan) was used as the secondary antibody. Color development was performed with 3,3'-diaminobenzidine (DAB, Dako) or HistoGreen (EUROBIO/ABCYS, Courtaboeuf, France). All images were obtained using an Olympus BX53 microscope (Olympus, Japan; objective lens PlanApo N 2X and U PlanApo 4X and 10X) and a DP22/U-TV0.5XC camera.

Double staining IHC with primary antibodies raised in the same species. We performed double staining for $\alpha \mathrm{SMA}$ and AE1/AE3. DAB was used to stain $\alpha$ SMA in the first step, and HistoGreen was used to stain AE1/AE3 in the second step. Because both antibodies are mouse monoclonal, high-temperature TE buffer was used to inactivate the anti- $\alpha$ SMA antibody after DAB staining.

Semiquantitative imaging analysis of the fibrotic and $\alpha S M A$ area indices in tumors. The entire tumor mass of each specimen was digitized using the microscope and camera described above. Based on previous studies $(15,32)$, we analyzed the images using ImageJ 1.50i (http://rsb.info.nih. gov/ij/) and the color deconvolution plugin (http://imagej. net/Colour_Deconvolution) for ImageJ and Fiji to implement staining separation via the method of Ruifrok and Johnston (33). Fibrosis was detected as a light green color in Elastica-Masson staining. The light-green positive area was extracted with the color deconvolution plugin (vector: Feulgen Light Green), and the area was converted to black (threshold: Upper cutoff, 214; lower cutoff, 0). After this processing, most of the remaining pixels in the image were those originally stained in light green, and we calculated the total area occupied by these pixels. This area was divided by the entire tumor area, and the extent of fibrosis (fibrotic area index) was determined as previously described (15). To obtain the $\alpha$ SMA-positive area ( $\alpha$ SMA area index) and AE1/AE3-positive area in the entire tumor, we used the same method used to determine the fibrotic area index (vector: $\mathrm{H}$ DAB; threshold: Upper cutoff, 200; lower cutoff, 0). Most of the cases had the reactive pleural fibrosis with mesothelioma cell invasion. For the fibrotic and $\alpha$ SMA area indices, we evaluated the entire tumor mass including pleural fibrosis since invasion to parietal and visceral pleura is a common pattern of mesothelioma invasion (2) and since the fibrosis 
A

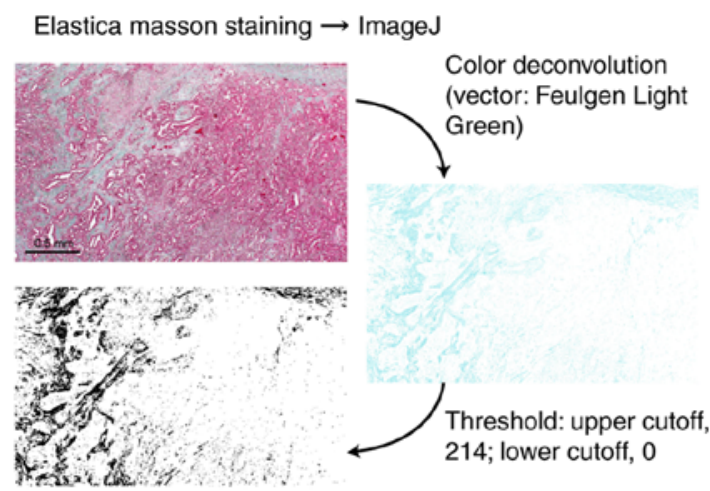

C $\alpha$ SMA (DAB)+AE1/AE3 (HistoGreen) $\rightarrow$ ImageJ

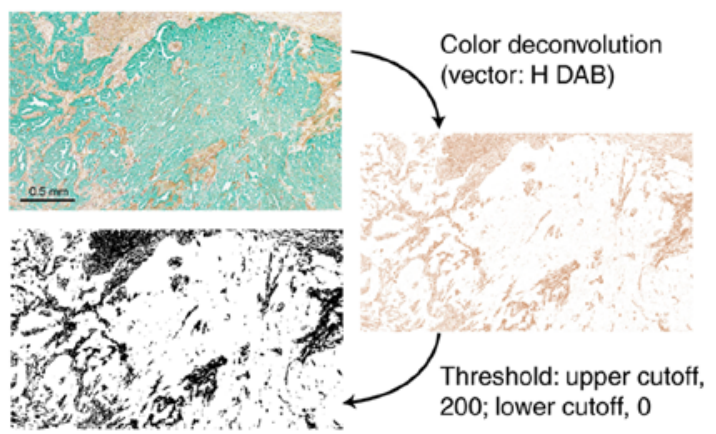

E

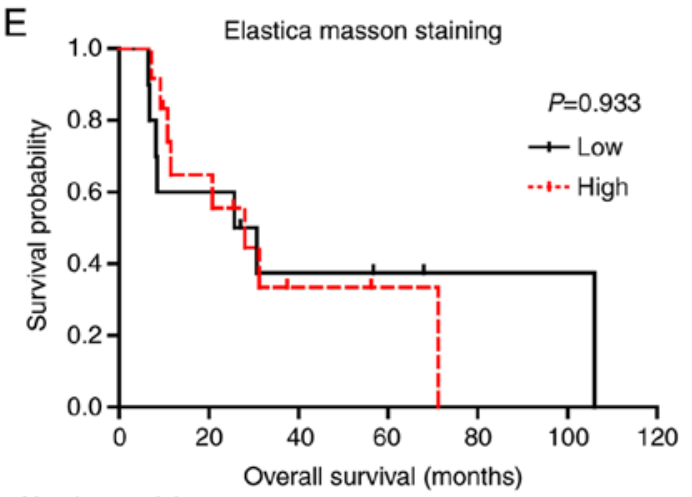

Number at risk

$\begin{array}{llllllll}\text { Low } & 10 & 6 & 3 & 2 & 1 & 1 & 0 \\ \text { High } & 12 & 7 & 2 & 1 & 0 & 0 & 0\end{array}$

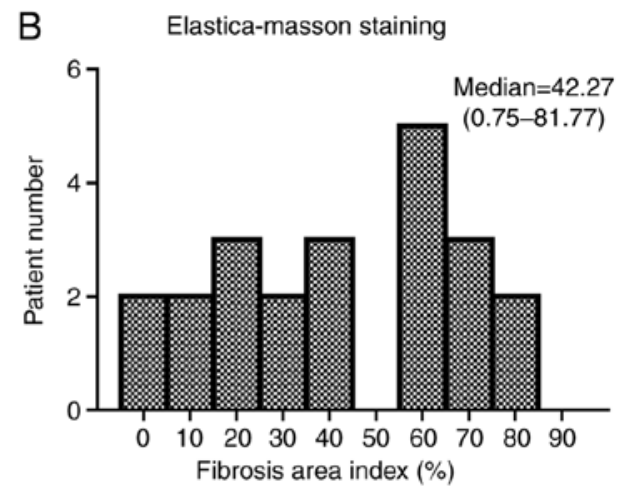

D

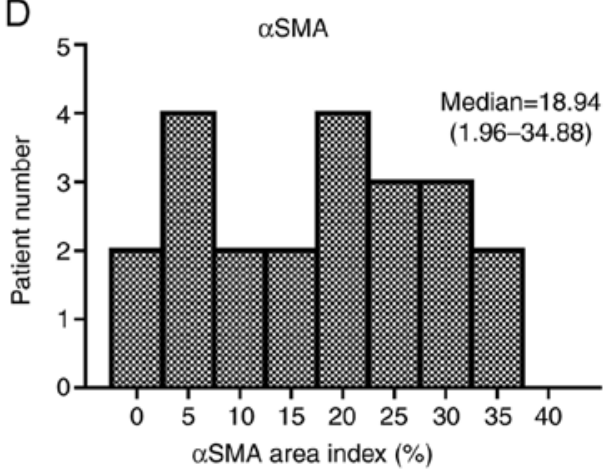

$\mathrm{F}$

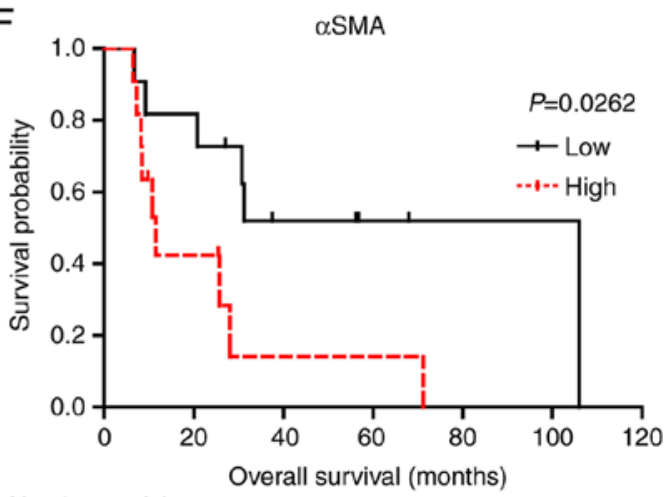

Number at risk

$\begin{array}{llllllll}\text { Low } & 11 & 9 & 4 & 2 & 1 & 1 & 0 \\ \text { High } & 11 & 4 & 1 & 1 & 0 & 0 & 0\end{array}$

Figure 1. Fibrotic and $\alpha$ SMA area indices in mesothelioma. (A) Calculation of the fibrotic area index using ImageJ. The image was obtained from mesothelioma in pleural fibrosis. (B) Analysis of Elastica-Masson staining. Fibrotic area indices for all cases are plotted as a histogram. (C) Calculation of the $\alpha$ SMA area index using ImageJ. DAB solution was used to stain $\alpha$ SMA, and HistoGreen was used to stain AE1/AE3. Mesothelioma cells were positive for AE1/AE3. The image was obtained from mesothelioma in pleural fibrosis. (D) Analysis of $\alpha$ SMA staining. The $\alpha$ SMA area indices for all cases are plotted as a histogram. (E) Fibrotic area index and patient prognosis based on Kaplan-Meier survival curves. There were no significant differences in prognosis based on the fibrotic area index (low, $<40 \%$; high, $\geq 40 \%$ ). (F) $\alpha$ SMA expression and patient prognosis based on Kaplan-Meier survival curves. There was a significant difference $(\mathrm{P}=0.0262)$ in prognosis based on the $\alpha$ SMA area index (low, $<20 \%$; high, $\geq 20 \%)$. $\alpha$ SMA, $\alpha$-smooth muscle actin; DAB, 3,3'-diaminobenzidine.

can contribute to mesothelioma progression. To minimize the influence of tumor heterogeneity, all the fields in the entire tumor were evaluated. The average of each entire tumor mass area was $141.7 \mathrm{~mm}^{2}$. We used a $2 \mathrm{x}$ objective lens and the average fields of view for each tumor mass was 7.6.

Semiquantitative analysis of CTGF expression in tumors. We used the entire tumor mass of each specimen. The CTGF immunostaining intensity in mesothelioma cells and CAFs was assessed as follows: 0, negative; 1 , weak; 2 , moderate; and 3 , strong. In addition, the $\mathrm{H}$-score for CTGF (CTGF score) was calculated using the following formula: [1x (\% of cells with an intensity of 1$)+2 x(\%$ of cells with an intensity of 2$)+3 x$ (\% of cells with an intensity of 3$)](34,35)$. All the fields were evaluated by a registered pathologist (YO). The average of each entire tumor mass area was $141.7 \mathrm{~mm}^{2}$. We used a $4 \mathrm{x}$ objective lens and the average fields of view for each tumor mass was 24.4. For the Kaplan-Meier survival curve, the CTGF score for mesothelioma cells and CAFs was modified by the tumor area (AE1/AE3-positive area) and stromal area ( $\alpha$ SMA-positive area) as follows: (CTGF score $x$ AE1/AE3 or $\alpha$ SMA area index). Using these modified CTGF scores, the patients were divided into two groups (low or high). 
Table I. Fibrotic area index and clinicopathological features of the mesothelioma cases.

\begin{tabular}{|c|c|c|c|}
\hline Characteristics & Low & High & P-value \\
\hline Age (years) & & & 0.670 \\
\hline$<65$ & 6 & 5 & \\
\hline$\geq 65$ & 4 & 7 & \\
\hline Sex & & & 0.571 \\
\hline Male & 8 & 11 & \\
\hline Female & 2 & 1 & \\
\hline Pathological invasion & & & 0.074 \\
\hline $\mathrm{pT} 1$ or $\mathrm{pT} 2$ & 6 & 2 & \\
\hline pT3 or pT4 & 4 & 10 & \\
\hline Lymph node metastasis & & & 0.391 \\
\hline $\mathrm{pNO}$ & 4 & 8 & \\
\hline $\mathrm{pN} 1$ or $\mathrm{pN} 2$ & 6 & 4 & \\
\hline Stage & & & 0.624 \\
\hline I or II & 3 & 2 & \\
\hline III or IV & 7 & 10 & \\
\hline Neoadjuvant chemotherapy & & & 0.348 \\
\hline Absent & 4 & 2 & \\
\hline Present & 6 & 10 & \\
\hline Chemosensitivity & & & $>0.999$ \\
\hline Grade 0 or $1 \mathrm{a}$ & 4 & 7 & \\
\hline Grade $1 b$ or 2 & 2 & 3 & \\
\hline
\end{tabular}

The fibrotic area index was calculated by Elastica-Masson staining. The index did not correlate with clinicopathological features. Cisplatin and pemetrexed were administered to 16 out of 22 patients as neoadjuvant chemotherapy. One patient was readministered carboplatin and pemetrexed due to cisplatin-induced vomiting. Fibrotic area index: Low, $<40 \%$; high, $\geq 40 \%$.

In situ hybridization of Meflin. In situ hybridization (ISH) analysis was performed using four-micron-thick formalin-fixed and paraffin-embedded human tissue sections with the RNAscope technology (RNAscope 2.5 HD Detection Kit; Advanced Cell Diagnostics) and a custom-designed probe of human Meflin according to the manufacturer's instructions, as previously described $(28,29)$. Briefly, tissue sections were baked in a dry oven (HybEZ II Hybridization System; Advanced Cell Diagnostics) at $60^{\circ} \mathrm{C}$ for $1 \mathrm{~h}$, deparaffinized, and incubated with $\mathrm{H}_{2} \mathrm{O}_{2}$ solution (Pretreat 1 buffer) for $10 \mathrm{~min}$ at room temperature. The slides were boiled in target retrieval solution (Pretreat 2 buffer) for $30 \mathrm{~min}$, incubated with protease solution (Pretreat 3 buffer) for $30 \mathrm{~min}$ at $40^{\circ} \mathrm{C}$, incubated with the probe for $2 \mathrm{~h}$ at $40^{\circ} \mathrm{C}$, and then successively incubated with Amp1 to 6 reagents. Staining was visualized with DAB, followed by counterstaining with hematoxylin. The RNAscope probe was as follows: Human Meflin (ISLR) (NM_005545.3, region 275-1322; cat. no. 455481). The slides were evaluated, as previously described (29).

ImageJ software was used for obtaining the merged image of $\alpha$ SMA + AE1/AE3 and Meflin. To detect the Meflin-positive area, we used the same method used to determine the $\alpha \mathrm{SMA}$
Table II. $\alpha$ SMA area index and clinicopathological features of the mesothelioma cases.

\begin{tabular}{|c|c|c|c|}
\hline Characteristics & Low & High & P-value \\
\hline Age (years) & & & $>0.999$ \\
\hline$<65$ & 6 & 5 & \\
\hline$\geq 65$ & 5 & 6 & \\
\hline Sex & & & $>0.999$ \\
\hline Male & 10 & 9 & \\
\hline Female & 1 & 2 & \\
\hline Pathological invasion & & & 0.183 \\
\hline $\mathrm{pT} 1$ or $\mathrm{pT} 2$ & 6 & 2 & \\
\hline pT3 or pT4 & 5 & 9 & \\
\hline Lymph node metastasis & & & 0.670 \\
\hline pN0 & 7 & 5 & \\
\hline $\mathrm{pN} 1$ or $\mathrm{pN} 2$ & 4 & 6 & \\
\hline Stage & & & 0.311 \\
\hline I or II & 4 & 1 & \\
\hline III or IV & 7 & 10 & \\
\hline Neoadjuvant chemotherapy & & & 0.635 \\
\hline Absent & 4 & 2 & \\
\hline Present & 7 & 9 & \\
\hline Chemosensitivity & & & 0.596 \\
\hline Grade 0 or $1 \mathrm{a}$ & 4 & 7 & \\
\hline Grade $1 b$ or 2 & 3 & 2 & \\
\hline
\end{tabular}

The aSMA area index was calculated by the immunohistochemical staining of $\alpha$ SMA. The index did not correlate with clinicopathological features. Cisplatin and pemetrexed were administered to 16 out of 22 patients as neoadjuvant chemotherapy. One patient was readministered carboplatin and pemetrexed due to cisplatin-induced vomiting. $\alpha \mathrm{SMA}$ area index: Low, $<20 \%$; high, $\geq 20 \%$. $\alpha$ SMA, $\alpha$-smooth muscle actin.

area index (vector: H DAB; threshold: Upper cutoff, 190; lower cutoff, 0 ). The Meflin-positive area was indicated by red color and merged with $\alpha \mathrm{SMA}+\mathrm{AE} 1 / \mathrm{AE} 3$ using Image Calculator.

Statistical analysis. The data were analyzed using GraphPad Prism 5 (GraphPad Software). The correlation between the expression of CAF markers and various clinicopathological features was analyzed by the Fisher's exact test. Correlation analysis was performed using non-parametric method (Spearman's rank correlation coefficient). The overall survival rate was calculated according to the Kaplan-Meier method and compared using the Log-rank test if not indicated otherwise. Gehan-Breslow-Wilcoxon test was used if crossover between the groups was observed at late timepoints. A P-value of $<0.05$ was considered as indicative of statistical significance.

\section{Results}

aSMA-positive $\left(\alpha S M A^{+}\right)$CAFs correlate with patient prognosis. Through H\&E, IHC staining of $\alpha \mathrm{SMA}+\mathrm{AE} 1 / \mathrm{AE} 3$, and Elastica-Masson staining, a high extent of fibrosis was observed in the reactive pleura present adjacent to mesothelioma 

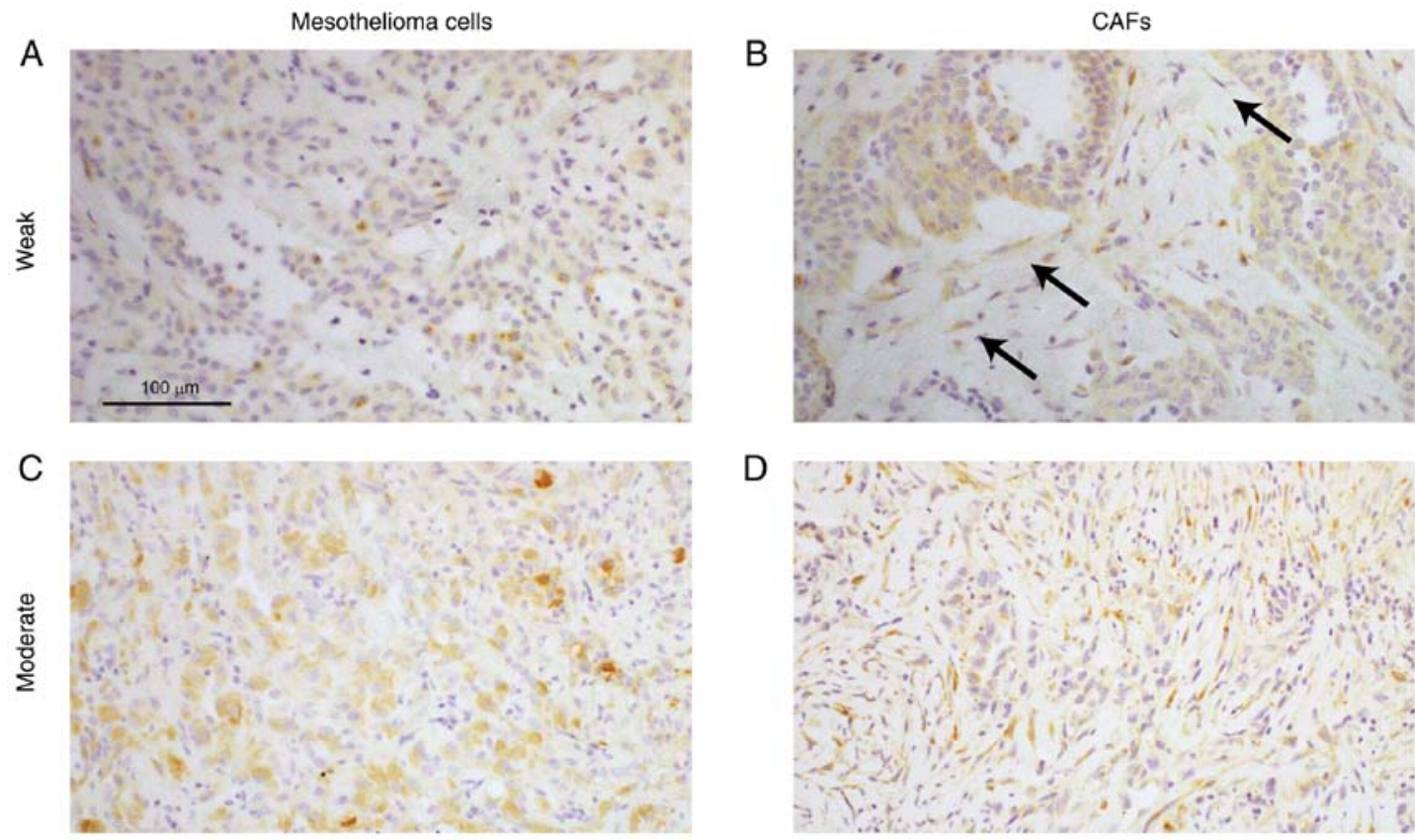

D
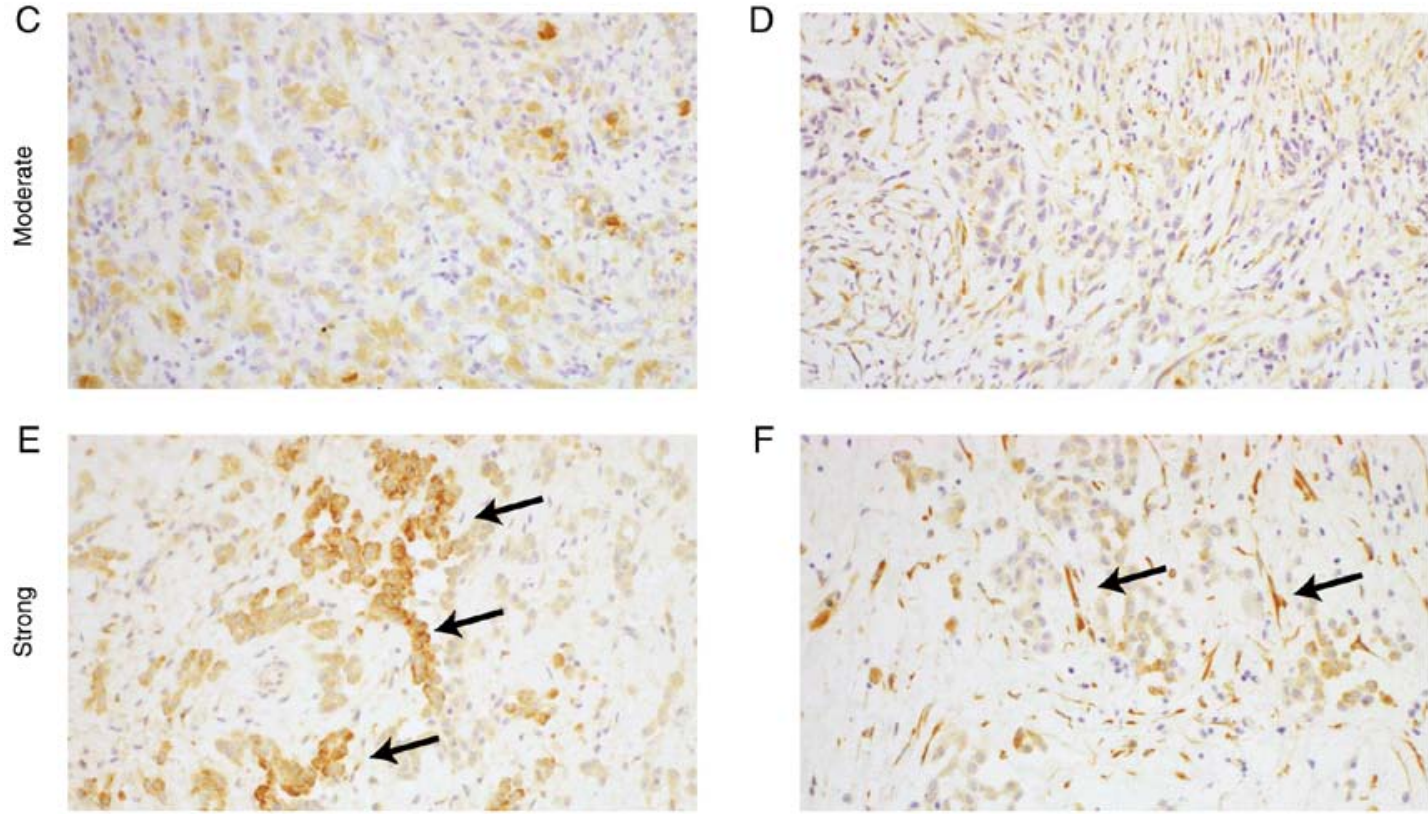

Figure 2. Immunohistochemical staining of CTGF. Both mesothelioma cells and CAFs were stained for CTGF. (A and B) Weak staining; score=1. The arrows in B indicate CAFs. (C and D) Moderate staining; score=2. ( $E$ and F) Strong staining; score=3. The arrows in E indicate mesothelioma cells. The arrows in F indicate CAFs. All images are shown at the same magnification. CAFs, cancer-associated fibroblasts; CTGF, connective tissue growth factor.

(Fig. S1). To confirm the correlation between fibrosis or CAFs and mesothelioma patient features, we first evaluated the density of fibrosis and the presence of CAFs expressing $\alpha$ SMA using paraffin-embedded sections of mesothelioma samples. Although epithelioid mesothelioma is positive for AE1/AE3 $(2,36)$, approximately $20 \%$ of sarcomatoid mesothelioma is negative for AE1/AE3 (37,38). In addition, it has been reported that reactive spindle cells can be positive for AE1/AE3 in sarcomatoid mesothelioma (39). Although the H\&E and IHC staining distinguished mesothelioma cells from CAFs in epithelioid mesothelioma (Fig. S2), our cases of biphasic or sarcomatoid mesothelioma contained cells for which it was difficult to clarify whether they were mesothelioma cells or CAFs. Thus, we excluded cases of biphasic and sarcomatoid mesothelioma. The area indices of fibrosis and $\alpha$ SMA were quantified based on color deconvolution (Fig. 1A-D), and the clinicopathological findings are summarized (Tables I and II). The indices of fibrosis and $\alpha$ SMA did not correlate with the clinicopathological features. In addition, no significant differences in overall survival were found based on the fibrotic area index (Fig. 1E). However, a significant difference ( $\mathrm{P}=0.0262$ ) was found based on the $\alpha \mathrm{SMA}$ area index (Fig. 1F).

CTGF-positive $\left(\mathrm{CTGF}^{+}\right) \mathrm{CAFs}$ correlate with mesothelioma cell proliferation and patient prognosis. To confirm CTGF as a prognostic factor and potential targets, we next evaluated the expression of CTGF and Ki-67 using paraffin-embedded sections. Mesothelial cells, which are nontumorous, did not express CTGF (Fig. S3), whereas obvious CTGF expression was observed in both mesothelioma cells and CAFs (Figs. 2A-F and S2). Biphasic and sarcomatoid mesotheliomas were excluded after the IHC staining results were examined, as described above. Heterogeneity in CTGF expression was observed in both mesothelioma cells and CAFs. We therefore adopted a semiquantitative scoring system (CTGF score; see Materials and methods) to quantify the expression of CTGF in each tumor sample (Fig. 3A and B) and compared these scores with the numbers of Ki-67-positive cells (Ki-67 index). The CTGF score for CAFs but not that of mesothelioma cells was correlated with the Ki-67 index for mesothelioma cells (Fig. 3C and D). The CTGF score for CAFs was also correlated with the $\alpha$ SMA area index, while that for mesothelioma cells was not (Fig. 3E and F). No significant correlations were found between CTGF expression in mesothelioma cells and patient prognosis (Fig. 3G). However, CTGF expression in CAFs correlated with poor prognosis (Fig. 3H). Notably, the clinicopathological features (pathological invasion, lymph node metastasis, and stage) and sensitivities to neoadjuvant chemotherapy of the examined mesothelioma cases did not 

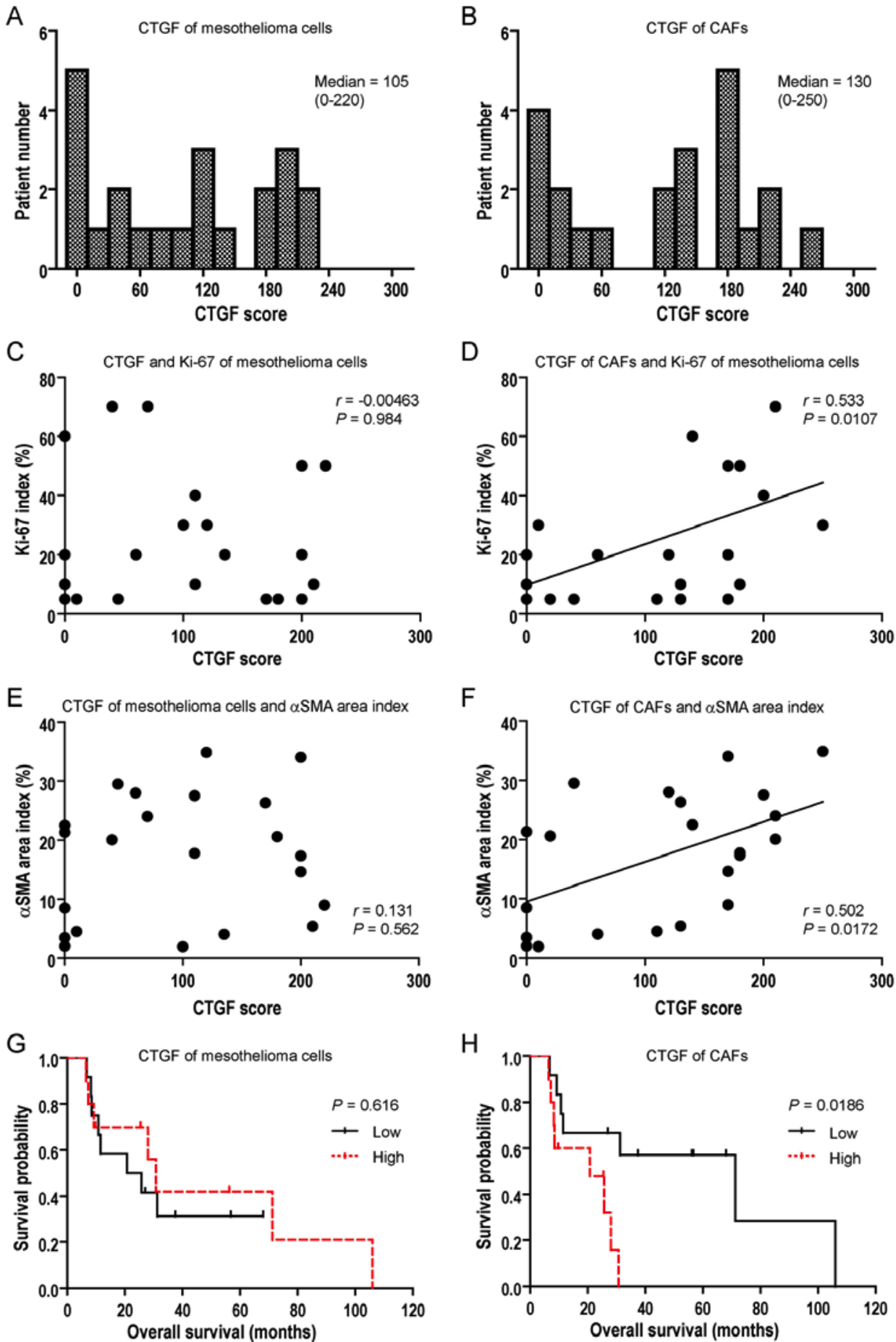

Number at risk

Number at risk

\begin{tabular}{|c|c|c|c|c|c|c|c|c|c|c|c|c|c|}
\hline Low 12 & 7 & 2 & 1 & 0 & 0 & 0 & Low & 12 & 8 & 5 & 3 & 1 & 1 \\
\hline High 10 & 6 & 3 & 2 & 1 & 1 & 0 & High & 10 & 5 & 0 & 0 & 0 & 0 \\
\hline
\end{tabular}

Figure 3. CTGF expression in CAFs correlates with mesothelioma patient prognosis. (A and B) Analysis of immunohistochemical staining of CTGF. The H-score of CTGF (CTGF score) for all cases is plotted as a histogram. (C) CTGF expression in mesothelioma cells and the Ki-67 index, indicating no significant differences. (D) CTGF expression in CAFs and the Ki-67 index, indicating a positive correlation ( $\mathrm{r}=0.533$, $\mathrm{P}=0.0107$; Spearman's correlation test). (E) CTGF expression in mesothelioma cells and the $\alpha$ SMA area index, indicating no significant differences. (F) CTGF expression in CAFs and the $\alpha$ SMA area index, indicating a positive correlation ( $\mathrm{r}=0.502, \mathrm{P}=0.0172$; Spearman's correlation test). (G) CTGF expression in mesothelioma cells and patient prognosis based on Kaplan-Meier survival curves. CTGF scores were modified by the AE1/AE3 area index values. There were no significant differences in prognosis based on modified CTGF scores (low, $<10$; high, $\geq 10$ ). (H) CTGF expression in CAFs and patient prognosis based on Kaplan-Meier survival curves. CTGF scores were modified by the $\alpha$ SMA area index values. There was a significant difference $(\mathrm{P}=0.0186)$ in prognosis based on modified CTGF score (low, $<30$; high, $\geq 30$ ). $\alpha$ SMA, $\alpha$-smooth muscle actin; CAFs, cancer-associated fibroblasts; CTGF, connective tissue growth factor.

correlate with CTGF expression in either mesothelioma cells or CAFs (Tables III and IV), suggesting that CTGF in CAFs could be used as a marker that specifically predicts patient prognosis. 
Table III. CTGF in mesothelioma cells and clinicopathological features of the mesothelioma cases.

\begin{tabular}{lccc}
\hline Characteristics & Low & High & P-value \\
\hline Age (years) & & & 0.670 \\
$<65$ & 7 & 4 & \\
$\geq 65$ & 5 & 6 & \\
Sex & & & 0.571 \\
$\quad$ Male & 11 & 8 & \\
Female & 1 & 2 & \\
Pathological invasion & & & 0.675 \\
pT1 or pT2 & 5 & 3 & \\
pT3 or pT4 & 7 & 7 & \\
Lymph node metastasis & & & 0.691 \\
pN0 & 6 & 6 & \\
pN1 or pN2 & 6 & 4 & \\
Stage & & & $>0.999$ \\
I or II & 3 & 2 & \\
III or IV & 9 & 8 & \\
Neoadjuvant chemotherapy & & & $>0.999$ \\
$\quad$ Absent & 3 & 3 & \\
Present & 9 & 7 & \\
Chemosensitivity & & & 0.308 \\
$\quad$ Grade 0 or 1a & 5 & 6 & \\
Grade 1b or 2 & 4 & 1 & \\
$\quad$
\end{tabular}

The modified CTGF score of mesothelioma cells did not correlate with clinicopathological features. Cisplatin and pemetrexed were administered to 16 out of 22 patients as neoadjuvant chemotherapy. One patient was readministered carboplatin and pemetrexed due to cisplatin-induced vomiting. Modified CTGF score: Low, <10; high, $\geq 10$. CTGF, connective tissue growth factor.

Meflin as a potential marker for mesothelioma patient prognosis. We next investigated Meflin expression by RNA ISH using paraffin-embedded sections. Mesothelial cells, which are nontumorous, did not express Meflin (Fig. S2), while CAFs expressed Meflin (Fig. 4A and B). More Meflin ${ }^{+}$ CAFs were observed in the $\alpha$ SMA-negative area than in the $\alpha$ SMA-positive area. Meflin expression did not correlate with the clinicopathological features (Table V). Additionally, no significant differences were found in patient prognosis according to Meflin expression (Fig. 4C).

\section{Discussion}

In the present study, we demonstrated that not only mesothelioma cells but also cancer-associated fibroblasts (CAFs) in mesothelioma express connective tissue growth factor (CTGF). CTGF expression in CAFs was found to be correlated with patient prognosis although CTGF expression in mesothelioma cells did not. The CTGF score for mesothelioma cells did not correlate with the Ki-67 index, but that for CAFs did. In addition, CTGF expression did not correlate with tumor stage. If a marker correlates with poor prognosis and tumor stage,
Table IV. CTGF in CAFs and clinicopathological features of the mesothelioma cases.

\begin{tabular}{lccc}
\hline Characteristics & Low & High & P-value \\
\hline Age (years) & & & 0.198 \\
$<65$ & 8 & 3 & \\
$\geq 65$ & 4 & 7 & \\
Sex & & & $>0.999$ \\
$\quad$ Male & 10 & 9 & \\
Female & 2 & 1 & \\
Pathological invasion & & & 0.675 \\
pT1 or pT2 & 5 & 3 & \\
pT3 or pT4 & 7 & 7 & \\
Lymph node metastasis & & & 0.691 \\
pN0 & 6 & 6 & \\
pN1 or pN2 & 6 & 4 & \\
Stage & & & $>0.999$ \\
I or II & 3 & 2 & \\
III or IV & 9 & 8 & \\
Neoadjuvant chemotherapy & & & 0.646 \\
$\quad$ Absent & 4 & 2 & \\
$\quad$ Present & 8 & 8 & \\
Chemosensitivity & & & $>0.999$ \\
$\quad$ Grade 0 or 1a & 6 & 5 & \\
Grade 1b or 2 & 2 & 3 & \\
$\quad$
\end{tabular}

The modified CTGF score of CAFs did not correlate with clinicopathological features. Cisplatin and Pemetrexed were administered to 16 out of 22 patients as neoadjuvant chemotherapy. One patient was readministered carboplatin and pemetrexed due to cisplatin-induced vomiting. Modified CTGF score: Low, <30; high, $\geq 30$. CAFs, cancer-associated fibroblasts; CTGF, connective tissue growth factor.

it is possible that the correlation is driven by tumor stage. In other words, the marker is highly expressed in advanced stage tumors, which results in a correlation of marker expression with poor prognosis. In the present study, CTGF expression was found to be correlated with poor prognosis after surgery irrespective of the tumor stage diagnosed at surgery. Therefore, CTGF-positive $\left(\mathrm{CTGF}^{+}\right)$CAFs are directly correlated with tumor malignancy/progression and CTGF may be a molecular target for this disease.

Using tissue or serum samples, previous studies have revealed that sarcomatoid mesothelioma expresses higher levels of CTGF than the epithelioid subtype $(13,40)$. In another study, however, all human mesothelioma cell lines expressed CTGF irrespective of histological subtype (15). This apparent inconsistency can be explained by the results of the present study, that is, based on CTGF expression by CAFs in vivo. Cells of sarcomatoid mesothelioma are commonly spindle-shaped and accompanied by proliferating nonneoplastic CAFs, making it difficult to distinguish between these two cell types. Moreover, CTGF-specific monoclonal antibody (FG-3019, pamrevlumab) was reported to exhibit little effect on cancer cell proliferation in conventional 

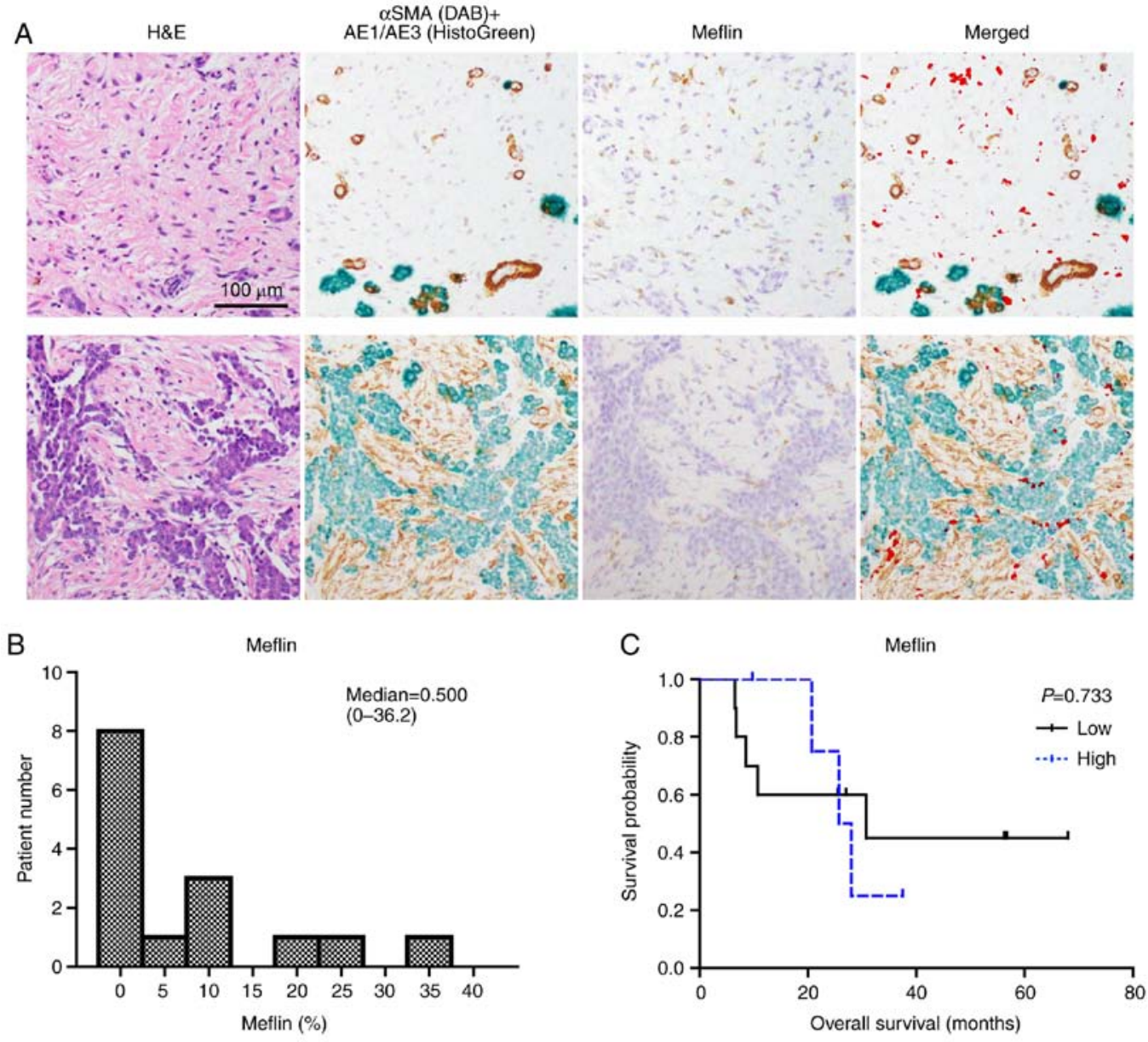

$\begin{array}{lccccc}\text { Number at risk } & & & & \\ \text { Low } 10 & 6 & 3 & 1 & 0 \\ \text { High } & 5 & 4 & 0 & 0 & 0\end{array}$

Figure 4. Meflin expression in mesothelioma. (A) RNA ISH of Meflin. DAB solution was used to stain $\alpha$ SMA, and HistoGreen was used to stain AE1/AE3. Mesothelioma cells were positive for AE1/AE3. CAFs and vessels were positive for $\alpha$ SMA. More Meflin-positive (Meflin ${ }^{+}$CAFs were observed in the $\alpha$ SMA-negative area (top), which is the invasive front of mesothelioma. Less Meflin ${ }^{+}$CAFs were observed in the $\alpha$ SMA-high area (bottom), which is the proximal side of mesothelioma. The merged images of $\alpha \mathrm{SMA}+\mathrm{AE} 1 / \mathrm{AE} 3$ and Meflin were obtained using ImageJ software. Meflin-positive area is indicated by red color in the merged images. All images are shown at the same magnification. (B) Analysis of RNA ISH data for Meflin. The proportions of the Meflin ${ }^{+}$ CAFs for all cases are plotted as a histogram. (C) Meflin expression in CAFs and patient prognosis based on Kaplan-Meier survival curves. There was no significant difference in prognosis based on the proportions of the Meflin ${ }^{+} \mathrm{CAFs}(\mathrm{low},<10 \%$; high, $\geq 10 \%$ ). Gehan-Breslow-Wilcoxon test was used for analysis $\alpha$ SMA, $\alpha$-smooth muscle actin; CAFs, cancer-associated fibroblasts; H\&E, hematoxylin and eosin; ISH, in situ hybridization; Meflin, mesenchymal stromal cell- and fibroblast-expressing Linx paralogue.

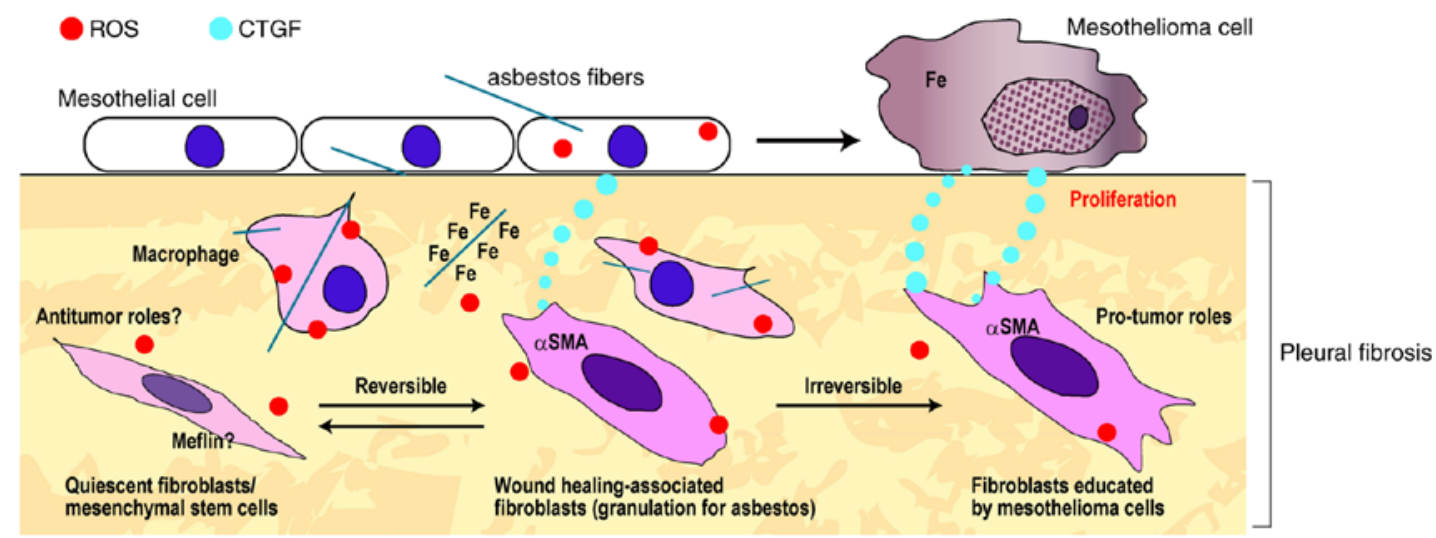

Figure 5. Fibroblasts in mesothelial carcinogenesis. Previous studies suggest the existence of three phenotypes of fibroblasts: Quiescent, wound healing-associated, and mesothelioma cell-educated. Asbestos fibers, which contain iron as a component, can directly induce reactive oxygen species (ROS) generation via catalysis of the Fenton reaction by iron on the surface. Macrophages phagocytose asbestos fibers and form granulomas. These macrophages can also produce ROS. ROS can induce quiescent fibroblasts to differentiate into myofibroblasts. These $\alpha \mathrm{SMA}^{+}$fibroblasts can contribute to carcinogenesis by secreting CTGF and cytokines. Fibroblasts educated by mesothelioma cells express CTGF and have protumorigenic roles. Meflin ${ }^{+}$fibroblasts may have antitumorigenic roles. $\alpha$ SMA, $\alpha$-smooth muscle actin; CTGF, connective tissue growth factor; Meflin, mesenchymal stromal cell- and fibroblast-expressing Linx paralogue. 
Table V. Meflin and clinicopathological features of the mesothelioma cases.

\begin{tabular}{|c|c|c|c|}
\hline Characteristics & Low & High & P-value \\
\hline Age (years) & & & $>0.999$ \\
\hline$<65$ & 4 & 2 & \\
\hline$\geq 65$ & 6 & 3 & \\
\hline Sex & & & 0.333 \\
\hline Male & 10 & 4 & \\
\hline Female & 0 & 1 & \\
\hline Pathological invasion & & & $>0.999$ \\
\hline $\mathrm{pT} 1$ or $\mathrm{pT} 2$ & 4 & 2 & \\
\hline pT3 or pT4 & 6 & 3 & \\
\hline Lymph node metastasis & & & 0.580 \\
\hline pNO & 5 & 4 & \\
\hline $\mathrm{pN} 1$ or $\mathrm{pN} 2$ & 5 & 1 & \\
\hline Stage & & & $>0.999$ \\
\hline I or II & 3 & 1 & \\
\hline III or IV & 7 & 4 & \\
\hline Neoadjuvant chemotherapy & & & 0.600 \\
\hline Absent & 4 & 1 & \\
\hline Present & 6 & 4 & \\
\hline Chemosensitivity & & & $>0.999$ \\
\hline Grade 0 or $1 \mathrm{a}$ & 4 & 3 & \\
\hline Grade $1 \mathrm{~b}$ or 2 & 2 & 1 & \\
\hline
\end{tabular}

Meflin did not correlate with clinicopathological features. Cisplatin and pemetrexed were administered to 10 out of 15 patients as neoadjuvant chemotherapy. Meflin: Low, $<10 \%$; high, $\geq 10 \%$. Meflin, mesenchymal stromal cell- and fibroblast-expressing Linx paralogue.

2-dimensional cell culture in vitro, whereas it strongly inhibited cancer growth in vivo $(15,41-43)$. These results can also be due to the existence of $\mathrm{CTGF}^{+} \mathrm{CAFs}$.

In the present study, the $\alpha$ SMA area index was found to be correlated with prognosis, as shown previously (16-20,44-47). Although fibrosis in mesothelioma is distinctive from that in other tumors, it is suggested that $\alpha \mathrm{SMA}^{+}$fibroblasts correlate with mesothelioma growth. Inhaled asbestos can first result in benign pleural fibrosis and then in mesothelioma $(1,2,11)$. Thus, mesothelioma tissues may exhibit substantial fibrosis from the precancerous lesion/early mesothelioma stage in situ, although tumor cells in other tumors involve fibroblasts and form stroma only when they invade. In addition, reactive oxygen species (ROS), playing a key molecular mechanism in mesothelial carcinogenesis (4-8), can activate quiescent fibroblasts to form myofibroblasts (18). Therefore, not all $\alpha \mathrm{SMA}^{+}$fibroblasts in mesotheliomas may be CAFs that are under the command of mesothelioma cells, as some of these cells may be wound healing-associated (related to granulation for asbestos) fibroblasts (Fig. 5). These myofibroblasts may also express CTGF, because we previously confirmed that normal fibroblasts can also express CTGF in vitro (15). These cells can contribute to carcinogenesis by secreting CTGF and cytokines.
For this study, we used immunohistochemistry (IHC) to demonstrate the roles of CAFs in mesothelioma progression as IHC rarely decreases the signal compared to immunofluorescence (IF). IHC made it possible to evaluate all of the specimens. In contrast, IF of $\alpha$ SMA/CTGF may be useful for studying the differentiation of CAFs from mesenchymal stem cells and for classifying CAFs $\left(\alpha \mathrm{SMA} \mathrm{CTGF}^{-}, \alpha \mathrm{SMA}^{+} / \mathrm{CTGF}^{-}\right.$, $\alpha \mathrm{SMA}^{-} / \mathrm{CTGF}^{+}$, and $\left.\alpha \mathrm{SMA}^{+} / \mathrm{CTGF}^{+}\right)$. We will perform such studies and develop the IF of $\alpha$ SMA/CTGF in the future.

A limitation in this study is that the eventual number of cases was not large. In our hospital, surgical cases of mesothelioma are rare because of the rareness of the disease and because the majority of cases were at the advanced stage at diagnosis. In the stroma, Meflin expression appeared positive where $\alpha$ SMA expression was negative in some lesions of the mesothelioma tissue samples. The present study did not elucidate whether Meflin correlates with patient survival. We were able to collect samples from only 15 patients as RNA ISH needs to be performed on tissue samples within five years of sample collection. CTGF expression did not correlated with sensitivities to neoadjuvant chemotherapy. This may be also because the number of cases is small for analysis. We will gather more samples to examine the expression of CAF markers ( $\alpha$ SMA, CTGF, and Meflin) and the correlations of CAFs and chemotherapy in the future.

In conclusion, $\mathrm{CTGF}^{+} \mathrm{CAFs}$ are important for mesothelioma growth and correlate with patient prognosis. Thus, these cells may be a potential target for drugs. Our previous study demonstrated that FG-3019 was effective for mesothelioma in a murine orthotopic implantation model, and the results of the present study suggest that FG-3019 targets CTGF ${ }^{+}$CAFs. Thus, whether FG-3019 has therapeutic effects in human mesothelioma patients warrants further investigation.

\section{Acknowledgements}

We thank Ms Tomomi Aoyama, Ms Naomi Tagami, and Dr Hideki Tsubouchi (Nagoya University) for technical assistance. YO was a recipient of the Takeda Science Foundation Fellowship (April 2014-March 2018).

\section{Funding}

The present study was supported in part by in part, from JST CREST (grant no. JPMJCR19H4) and JSPS Kakenhi (grant nos. JP17H04064, JP19H05462 and JP20H05502) to ST and by a Meidai Ishikai Funding 2016 to YO.

\section{Availability of data and materials}

Data and materials are available upon request to the corresponding author.

\section{Authors' contributions}

YO and ST designed the experiments. YO performed the experiments and data analysis, wrote the main manuscript text, and prepared the figures. AE, YT, KS, TI, HK, YMiz, YMiy, AH, SM, JS, KY, FI, YMo, NM, TF, KK and KY provided administrative, technical, and material support. All authors 
read and approved the manuscript and agree to be accountable for all aspects of the research in ensuring that the accuracy or integrity of any part of the work are appropriately investigated and resolved.

\section{Ethics approval and consent to participate}

Human mesothelioma tissues were obtained with informed patient consent at the time of surgery at Nagoya University Hospital (Nagoya, Japan). This study was carried out in accordance with the Helsinki Declaration for Human Research and approved by the Ethics Committee of Nagoya University Graduate School of Medicine (protocol no. 2017-0127).

\section{Patient consent for publication}

Not applicable.

\section{Competing interests}

The authors declare that they have no competing interests.

\section{References}

1. International Agency for Research on Cancer: WHO. Asbestos (chrysotile, amosite, crocidolite, tremolite, actinolite, and anthophyllite). IARC Monographs on the Evaluation of Carcinogenic Risks to Humans Volume 100C. A Review of Human Carcinogens; Part C: Arsenic. Metals, Fibres, and Dusts, Lyon, France, pp. 219-309, 2012.

2. Pavlisko EN and Sporn TA: Mesothelioma. Pathology of asbestos-associated diseases 3rd edn. Springer, Berlin, pp. 81-140, 2014

3. Rusch VW, Giroux D, Kennedy C, Ruffini E, Cangir AK, Rice D, Pass H, Asamura H, Waller D, Edwards J, et al: Initial analysis of the international association for the study of lung cancer mesothelioma database. J Thorac Oncol 7: 1631-1639, 2012.

4. Donaldson K, Murphy FA, Duffin R and Poland CA: Asbestos, carbon nanotubes and the pleural mesothelium: A review of the hypothesis regarding the role of long fibre retention in the parietal pleura, inflammation and mesothelioma. Part Fibre Toxicol 7: 5, 2010.

5. Nagai H and Toyokuni S: Biopersistent fiber-induced inflammation and carcinogenesis: Lessons learned from asbestos toward safety of fibrous nanomaterials. Arch Biochem Biophys 502: 1-7, 2010.

6. Chew SH and Toyokuni S: Malignant mesothelioma as an oxidative stress-induced cancer: An update. Free Radic Biol Med 86: 166-178, 2015.

7. Nagai $\mathrm{H}$ and Toyokuni S: Differences and similarities between carbon nanotubes and asbestos fibers during mesothelial carcinogenesis: Shedding light on fiber entry mechanism. Cancer Sci 103: 1378-1390, 2012

8. Toyokuni S: Iron addiction with ferroptosis-resistance in asbestos-induced mesothelial carcinogenesis: Toward the era of mesothelioma prevention. Free Radic Biol Med 133: 206-215, 2019.

9. Toyokuni S: Role of iron in carcinogenesis: Cancer as a ferrotoxic disease. Cancer Sci 100: 9-16, 2009.

10. Ohara Y, Chew SH, Shibata T, Okazaki Y, Yamashita K and Toyokuni S: Phlebotomy as a preventive measure for crocidolite-induced mesothelioma in male rats. Cancer Sci 109: 330-339, 2018.

11. Jiang L, Chew SH, Nakamura K, Ohara Y, Akatsuka S and Toyokuni S: Dual preventive benefits of iron elimination by desferal in asbestos-induced mesothelial carcinogenesis. Cancer Sci 107: 908-915, 2016.

12. Chew SH, Okazaki Y, Akatsuka S, Wang S, Jiang L, Ohara Y, Ito F, Saya H, Sekido Y and Toyokuni S: Rheostatic CD44 isoform expression and its association with oxidative stress in human malignant mesothelioma. Free Radic Biol Med 106: 91-99, 2017.
13. Jiang L, Yamashita Y, Chew SH, Akatsuka S, Ukai S, Wang S, Nagai H, Okazaki Y, Takahashi T and Toyokuni S: Connective tissue growth factor and $\beta$-catenin constitute an autocrine loop for activation in rat sarcomatoid mesothelioma. J Pathol 233: 402-414, 2014.

14. Wang S, Jiang L, Han Y, Chew SH, Ohara Y, Akatsuka S, Weng L, Kawaguchi K, Fukui T, Sekido Y, et al: Urokinase-type plasminogen activator receptor promotes proliferation and invasion with reduced cisplatin sensitivity in malignant mesothelioma. Oncotarget 7: 69565-69578, 2016.

15. Ohara Y, Chew SH, Misawa N, Wang S, Somiya D, Nakamura K, Kajiyama H, Kikkawa F, Tsuyuki Y, Jiang L, et al: Connective tissue growth factor-specific monoclonal antibody inhibits growth of malignant mesothelioma in an orthotopic mouse model. Oncotarget 9: 18494-18509, 2018.

16. Hanahan D and Weinberg RA: Hallmarks of cancer: The next generation. Cell 144: 646-674, 2011.

17. Orimo A and Weinberg RA: Stromal fibroblasts in cancer: A novel tumor-promoting cell type. Cell Cycle 5: 1597-1601, 2006.

18. Kalluri R: The biology and function of fibroblasts in cancer. Nat Rev Cancer 16: 582-598, 2016.

19. LeBleu VS and Kalluri R: A peek into cancer-associated fibroblasts: Origins, functions and translational impact. Dis Model Mech 11: dmm029447, 2018.

20. Öhlund D, Elyada E and Tuveson D: Fibroblast heterogeneity in the cancer wound. J Exp Med 211: 1503-1523, 2014.

21. Rhim AD, Oberstein PE, Thomas DH, Mirek ET, Palermo CF, Sastra SA, Dekleva EN, Saunders T, Becerra CP, Tattersall IW, et al: Stromal elements act to restrain, rather than support, pancreatic ductal adenocarcinoma. Cancer Cell 25: 735-747, 2014.

22. Sherman MH, Yu RT, Engle DD, Ding N, Atkins AR, Tiriac H, Collisson EA, Connor F, Van Dyke T, Kozlov S, et al: Vitamin D receptor-mediated stromal reprogramming suppresses pancreatitis and enhances pancreatic cancer therapy. Cell 159: 80-93, 2014.

23. Öhlund D, Handly-Santana A, Biffi G, Elyada E, Almeida AS, Ponz-Sarvise M, Corbo V, Oni TE, Hearn SA, Lee EJ, et al: Distinct populations of inflammatory fibroblasts and myofibroblasts in pancreatic cancer. J Exp Med 214: 579-596, 2017.

24. Raghu G, Scholand MB, de Andrade J, Lancaster L, Mageto Y, Goldin J, Brown KK, Flaherty KR, Wencel M, Wanger J, et al: FG-3019 anti-connective tissue growth factor monoclonal antibody: Results of an open-label clinical trial in idiopathic pulmonary fibrosis. Eur Respir J 47: 1481-1491, 2016.

25. Richeldi L, Fernández Pérez ER, Costabel U, Albera C, Lederer DJ, Flaherty KR, Ettinger N, Perez R, Scholand MB, Goldin J, et al: Pamrevlumab, an anti-connective tissue growth factor therapy, for idiopathic pulmonary fibrosis (PRAISE): A phase 2, randomised, double-blind, placebo-controlled trial. Lancet Respir Med 8: 25-33, 2020.

26. Picozzi VJ, Pipas JM, Koong AC, Giaccia AJ, Bahary N, Krishnamurthi SS, Lopez CD, O'Dwyer P, Modelska K, Carney M, et al: FG-3019, a human monoclonal antibody to connective tissue growth factor, combined with chemotherapy in patients with locally advanced or metastatic pancreatic ductal adenocarcinoma. J Cancer Clin Trials 2: 1, 2017.

27. Maeda K, Enomoto A, Hara A, Asai N, Kobayashi T, Horinouchi A, Maruyama S, Ishikawa Y, Nishiyama T, Kiyoi $\mathrm{H}$, et al: Identification of Meflin as a potential marker for mesenchymal stromal cells. Sci Rep 6: 22288, 2016.

28. Hara A, Kobayashi H, Asai N, Saito S, Higuchi T, Kato K, Okumura T, Bando YK, Takefuji M, Mizutani Y, et al: Roles of the mesenchymal stromal/stem cell marker Meflin in cardiac tissue repair and the development of diastolic dysfunction. Circ Res 125: 414-430, 2019.

29. Mizutani Y, Kobayashi H, Iida T, Asai N, Masamune A, Hara A, Esaki N, Ushida K, Mii S, Shiraki Y, et al: Meflin-positive cancer-associated fibroblasts inhibit pancreatic carcinogenesis. Cancer Res 79: 5367-5381, 2019.

30. Sobin LH, Gospodarowicz MK and Wittekind C: TNM Classification of Malignant Tumors (UICC), 7th edn. New York, NY: Wiley-Liss, 2009.

31. Chew SH, Okazaki Y, Nagai H, Misawa N, Akatsuka S, Yamashita K, Jiang L, Yamashita Y, Noguchi M, Hosoda K, et al: Cancer-promoting role of adipocytes in asbestos-induced mesothelial carcinogenesis through dysregulated adipocytokine production. Carcinogenesis 35: 164-172, 2014. 
32. Kato T, Noma K, Ohara T, Kashima H, Katsura Y, Sato $H$ Komoto $\mathrm{S}$, Katsube $\mathrm{R}$, Ninomiya $\mathrm{T}$, Tazawa $\mathrm{H}$, et al: Cancer-associated fibroblasts affect intratumoral $\mathrm{CD}^{+}$and FoxP3 $3^{+} \mathrm{T}$ cells via IL6 in the tumor microenvironment. Clin Cancer Res 24: 4820-4833, 2018.

33. Ruifrok AC and Johnston DA: Quantification of histochemical staining by color deconvolution. Anal Quant Cytol Histol 23: 291-299, 2001.

34. Boyle TA, Masago K, Ellison KE, Yatabe Y and Hirsch FR: ROS1 immunohistochemistry among major genotypes of non-small-cell lung cancer. Clin Lung Cancer 16: 106-111, 2015.

35. Detre S, Saclani Jotti G and Dowsett M: A 'quickscore' method for immunohistochemical semiquantitation: Validation for oestrogen receptor in breast carcinomas. J Clin Pathol 48: 876-878, 1995

36. Husain AN, Colby TV, Ordóñez NG, Allen TC, Attanoos RL, Beasley MB, Butnor KJ, Chirieac LR, Churg AM, Dacic S, et al: Guidelines for pathologic diagnosis of malignant mesothelioma 2017 update of the consensus statement from the international mesothelioma interest group. Arch Pathol Lab Med 142: 89-108, 2018.

37. Attanoos RL, Dojcinov SD, Webb R and Gibbs AR Anti-mesothelial markers in sarcomatoid mesothelioma and other spindle cell neoplasms. Histopathology 37: 224-231, 2000.

38. Chirieac LR, Pinkus GS, Pinkus JL, Godleski J, Sugarbaker DJ and Corson JM: The immunohistochemical characterization of sarcomatoid malignant mesothelioma of the pleura. Am J Cancer Res 1: 14-24, 2011

39. Churg A, Cagle P, Colby TV, Corson JM, Gibbs AR, Hammar S, Ordonez N, Roggli VL, Tazelaar HD, Travis WD, et al: The fake fat phenomenon in organizing pleuritis: A source of confusion with desmoplastic malignant mesotheliomas. Am J Surg Pathol 35: 1823-1829, 2011.

40. Fujii M, Toyoda T, Nakanishi H, Yatabe Y, Sato A, Matsudaira Y, Ito $\mathrm{H}$, Murakami $\mathrm{H}$, Kondo $\mathrm{Y}$, Kondo E, et al: TGF- $\beta$ synergizes with defects in the Hippo pathway to stimulate human malignant mesothelioma growth. J Exp Med 209: 479-494, 2012.

41. Dornhöfer N, Spong S, Bennewith K, Salim A, Klaus S, Kambham N, Wong C, Kaper F, Sutphin P, Nacamuli R, et al: Connective tissue growth factor-specific monoclonal antibody therapy inhibits pancreatic tumor growth and metastasis. Cancer Res 66: 5816-5827, 2006.
42. Neesse A, Frese KK, Bapiro TE, Nakagawa T, Sternlicht MD, Seeley TW, Pilarsky C, Jodrell DI, Spong SM and Tuveson DA: CTGF antagonism with mAb FG-3019 enhances chemotherapy response without increasing drug delivery in murine ductal pancreas cancer. Proc Natl Acad Sci USA 110: 12325-12330, 2013.

43. Finger EC, Cheng CF, Williams TR, Rankin EB, Bedogni B, Tachiki L, Spong S, Giaccia AJ and Powell MB: CTGF is a therapeutic target for metastatic melanoma. Oncogene 33: 1093-1100, 2014.

44. Sinn M, Denkert C, Striefler JK, Pelzer U, Stieler JM, Bahra M, Lohneis P, Dörken B, Oettle H, Riess H and Sinn BV: $\alpha$-Smooth muscle actin expression and desmoplastic stromal reaction in pancreatic cancer: Results from the CONKO-001 study. Br J Cancer 111: 1917-1923, 2014.

45. Underwood TJ, Hayden AL, Derouet M, Garcia E, Noble F, White MJ, Thirdborough S, Mead A,Clemons N, Mellone M, et al: Cancer-associated fibroblasts predict poor outcome and promote periostin-dependent invasion in oesophageal adenocarcinoma. J Pathol 235: 466-477, 2015.

46. Valach J, Fík Z, Strnad H, Chovanec M, Plzák J, Cada Z, Szabo P, Sáchová J, Hroudová M, Urbanová M, et al: Smooth muscle actin-expressing stromal fibroblasts in head and neck squamous cell carcinoma: Increased expression of galectin-1 and induction of poor prognosis factors. Int J Cancer 131: 2499-2508, 2012.

47. Fujita H, Ohuchida K, Mizumoto K, Nakata K, Yu J, Kayashima T, Cui L, Manabe T, Ohtsuka T and Tanaka M: Alpha-smooth muscle actin expressing stroma promotes an aggressive tumor biology in pancreatic ductal adenocarcinoma. Pancreas 39: 1254-1262, 2010.

This work is licensed under a Creative Common Attribution-NonCommercial-NoDerivatives 4.0 International (CC BY-NC-ND 4.0) License. 